Physics Vol. 2, No. 6, pp. 263-272, 1966. Physics Publishing Co. Printed in Great Britain.

\title{
SCALING LAWS FOR ISING MODELS NEAR $T_{c}{ }^{*}$
}

\author{
LEO P. KADANOFF ${ }^{\dagger}$ \\ Department of Physics, University of Illinois \\ Urbana, Illinois \\ (Received 3 February 1966)
}

\begin{abstract}
A model for describing the behavior of Ising models very near $T_{c}$ is introduced. The description is based upon dividing the Ising model into cells which are microscopically large but much smaller than the coherence length and then using the total magnetization within each cell as a collective variable. The resulting calculation serves as a partial justification for Widom's conjecture about the homogeneity of the free energy and at the same time gives his result $s v^{\prime}=\gamma^{\prime}+2 \beta$.
\end{abstract}

\section{Introduction}

IN a recent paper [1] Widom has discussed the consequences of the assumption that the free energy in a system near a phase transition of second order is a homogeneous function of parameters which describe the deviation from the critical point and has shown that this assumption leads to consequences which roughly agree with our present numerical information [2] about the behavior of such systems. Another paper by Widom [3] written at the same time explores the consequences of an apparently quite independent idea: that the behavior of the interface separating droplets of the "wrong phase" within a system just below a phase transition should be quite similar to the behavior of an interface separating a region of fluctuation in the order parameter from the surrounding medium [4]. Here again information is derived which agrees with numerical calculations and experiment [2].

Widom's ideas about interfaces are based upon physical plausibility arguments; his idea about the homogeneity of the singular part of the free energy is not given any very strong justification beyond the fact that it appears to work. In the present paper, the Ising model is analyzed in a manner which is designed to throw light upon how correlations between the order parameter in different regions of the lattice scale when the parameters describing the deviation from the critical point - in this case $T-T_{c}$ and the applied magnetic field - are changed. Widom' $s$ homogeneity condition upon the singular part of the free energy and some of his results for interfaces are then derived as a consequence of these scaling arguments based upon our model.

- This research was supported in part by the National science Foundation under grant NSF-GP 4937.

$\dagger$ A.P. Sloan Foundation Fellow. 
Although the argument is carried out in Ising model language, it is clear that the arguments could be generalized to other cases of second order phase transitions.

\section{Description of Model}

Consider an Ising Model in a weak magnetic field near $T_{c}$. This model can be described in terms of two parameters

$$
\varepsilon=\left(T-T_{c}\right) / T_{c}
$$

which measures the deviation from the critical temperature, and $h$, a dimensionless magnetic field defined so that flipping a single spin gives a change in magnetic energy $2 h / k T$. A full solution of the Ising model would be obtained if we knew $f(\varepsilon, h)$, the free energy per site in the presence of the magnetic field.

To get some feeling for the behavior of the Ising model, imagine that we divided the entire lattice into cells of $L$ lattice sites on a side. Then each cell in this $s$-dimensional lattice contains $L^{s}$ lattice sites. We take $L$ to be large, but much smaller than the coherence length, $\xi$, which describes the range of spin correlations, measured in lattice constants. Since $\xi$ goes to infinity at $\varepsilon=0$ and $h=0$, it is easy to find an $L$ which satisfies this criterion.

To zeroth order, each cell can be considered to be isolated from the others and from the external magnetic field. Then to zeroth order $f(\varepsilon, h)=f_{L}(\varepsilon)$ where $f_{L}(\varepsilon)$ is a free energy per site of a lattice of side $L$ in no magnetic field. Since the small size of the cell tends to eliminate the singularities from the phase transition, $f_{L}(\varepsilon)$ should be an analytic function of $\varepsilon$ but not of $L$, i.e.

$$
f_{L}(\varepsilon)=f_{L}^{(0)}+\varepsilon f_{L}^{(1)}+\varepsilon^{2} f_{L}^{(2)}+\ldots
$$

where $f_{L}^{0}, f^{\prime}{ }_{L}$ and $f_{L}{ }^{2}$ should not all be analytic functions of $L$ [4].

Next consider interactions of the cell $\alpha$ with the magnetic field. This gives a term in $-\dot{\beta} \mathscr{H}$ of the form

$$
h \sum_{\mathbf{r} \in \alpha} \sigma_{\mathbf{r}}
$$

The basic assumption of our analysis is that within each cell, the spins tend to line up so that they mostly point either up or down. That is,

$$
\sum_{\mathbf{r} \in \alpha} \sigma_{\mathbf{r}}=\mu_{\alpha}<\sigma>_{L} L^{s}
$$

where $\mu_{\alpha}$ is either plus one or minus one. The average spin, $\langle\sigma\rangle_{L}$, defined in (4) is given by

$$
\langle\sigma\rangle_{L}^{2}=\sum_{\mathbf{r} \in \alpha} \sum_{\mathbf{r}^{\prime} \in \alpha} \frac{\left\langle\sigma_{\mathbf{r}} \sigma_{\mathbf{r}}{ }^{\prime}\right\rangle}{L^{2} s}
$$

Because of the "small" size of the cell, this depends strongly on only $L$, not upon $\varepsilon$ or $h$. Thus, the interaction with the magnetic field takes the form of a term in $\exp [-\beta \mathscr{H}]$ of

$$
\exp \left(\sum_{\alpha} h \mu_{\alpha} L s<\sigma>_{L}\right)
$$


Next, consider the interaction among cells. The free energy will tend to be larger if the spins on neighboring cells are lined up. There will tend to be a smaller contribution if they are anti-parallel. Then, in net, this tends to make a contribution to the exponential $\exp [-\beta F]$

$$
\exp \sum_{\alpha, \beta}\left\{\mu_{\alpha} \mu_{\beta} \tilde{K}(\varepsilon, L)+f_{\mathrm{int}}(\varepsilon, L)\right\} .
$$

Here the sum extends over nearest neighbor cells, $\widetilde{K}+f_{\text {int }}$ gives the contribution to the free energy when neighboring cells are aligned and, $-\widetilde{K}+f_{\text {int }}$ gives the contribution to the free energy when they are out of step. Since the direct interactions between cells which produce $f_{\text {int }}$ occur within a distance which is very short compared to the coherence length, we assume that $f_{\text {int }}$ is, like $f_{L}(\varepsilon)$, a regular function of $\varepsilon$, but not necessarily a regular function of $L$. On the other hand, $\widetilde{K}$ is perhaps a somewhat more subtle beast. This describes the extra free energy that it costs to put two cells out of step. This involves, then, the rather delicate difference between the ways cells can match up when they are in step and when they are out of step. Nonetheless, it seems reasonable to assume that $\widetilde{K}(\varepsilon, L)$ is also a regular function of $\varepsilon$; but, we assert this with somewhat less confidence than our other statements relating to this model. In writing (7) we are asserting that the correlations among cells can be totally represented by these interactions among near neighbors and that there are no less direct interactions that we need include in (7) as long ranged interactions. This statement, together with the assertion that the cell can be represented by the double valued variable, $\mu_{\alpha}$, are the two basic assumptions of this model.

To find the correction to the zeroth order result (1), we sum the product of (6) and (7) over both possible orientations of each $\mu_{\alpha}$. This sum is, of course, just an Ising model calculation with coupling constant $\tilde{K}$ and effective magnetic field $\tilde{h}$. This then gives a contribution to the total free energy

$$
\sum_{\alpha} s f_{\text {int }}(\varepsilon, L)+f(\tilde{\varepsilon}, \tilde{h})
$$

where $\tilde{\varepsilon}$ measures the deviation of the new coupling constant from the critical value, and where, from (6) the effective magnetic field in the cell problem is

$$
\tilde{h}(h, L)=h\langle\sigma\rangle_{L} L^{s}
$$

Since the original Ising model problem has within it correlations over many cells, we assert that $\tilde{\varepsilon}$ and $\tilde{h}$ must be sufficiently small so that the new Ising model problem retains long-ranged correlations in $\left\langle\mu_{\alpha \mu_{\beta}}>\right.$. This requires $\tilde{\varepsilon} \ll 1, \tilde{h} \ll 1$.

Since there are $L^{s}$ sites per cell, equations (1) and (9) give the free energy as

$$
f(\varepsilon, h)=f_{L}(\varepsilon)+s L-s f_{\mathrm{int}}(\varepsilon, L)+L-s f(\tilde{\varepsilon}, \tilde{h}) .
$$

Equation (10) is one of the two basic relations in our analysis. The other is obtained by discussing the spin-spin correlation function $\left\langle\sigma_{\mathbf{r}} \sigma_{\mathbf{r}}{ }^{\prime}\right\rangle$ for the case in which the distance between spins $\left|\mathbf{r}-\mathbf{r}^{\prime}\right|$, as measured in lattice constants, is much larger than $L$. Then each $\sigma_{\mathbf{r}}$ can be replaced by the average of $\sigma_{r}$ over the cell in which it lies so that (4) may be used to rewrite the correlation function as

$$
\left\langle\sigma_{\mathbf{r}} \sigma_{\mathbf{r}}{ }^{\prime}\right\rangle=g\left(\varepsilon, h,\left|\mathbf{r}-\mathbf{r}^{\prime}\right|\right)=\langle\sigma\rangle_{L}{ }^{2}\left\langle\mu_{\alpha} \mu_{\alpha^{\prime}}\right\rangle=\langle\sigma\rangle_{L}{ }^{2} g\left(\tilde{\varepsilon}, \tilde{h},\left|\mathbf{r}-\mathbf{r}^{\prime}\right| / L\right) .
$$


The last line of (11) follows because the $\mu^{\prime} s$ are described by an Ising model with effective distance from $T_{c}, \tilde{\varepsilon}$, effective magnetic field $\tilde{h}$ and cell $L$ lattice constants long.

\section{Analysis of the Model}

The free energy is a singular function of $\varepsilon$. For example if the specific heat diverges as $\varepsilon^{-\alpha}\left[(-\varepsilon)^{-\alpha^{\prime}}\right]$ above [below] $T_{c}$, for small $\varepsilon$ and $h=0$,

$$
f(\varepsilon, 0)=\left\{\begin{array}{l}
f_{0}+f_{1} \varepsilon+f_{2} \varepsilon^{2-\alpha}+\ldots \text { for } T>T_{c} \\
f_{0}+f_{1} \varepsilon+f^{\prime}{ }_{2}(-\varepsilon)^{2-\alpha^{\prime}}+\ldots \text { for } T<T_{c}
\end{array}\right.
$$

In three dimensions (2) $0 \leqslant \alpha \leqslant 1,0 \leqslant \alpha^{\prime} \leqslant 1$. Another possible behavior has the specific heat above and/or below $T_{c}$ diverge logarithmically so that $\alpha$ and/or $\alpha^{\prime}$ are zero but

$$
f(\varepsilon, 0)=f_{0}+f_{1} \varepsilon+1 / 2 \varepsilon^{2}[B-A \log \varepsilon] \text { for } T>T_{c}
$$

and/or

$$
f(\varepsilon, 0)=f_{0}+f_{1} \varepsilon+1 / 2 \varepsilon^{2}\left[B^{\prime}-A^{\prime} \log |\varepsilon|\right] \text { for } T<T_{c}
$$

We first attack the case in which the $\alpha$ 's differ from zero and later consider the possibility of behaviors (12b) and/or (12c).

Consider first, at $h=0$, the terms in equation (10) which are singular in $\varepsilon$ and $\tilde{\varepsilon}$. If we equate these singular terms above $T_{c}$, we find

$$
\varepsilon^{2-\alpha}=[\tilde{\varepsilon}]^{2-\alpha} / L^{s} .
$$

It follows then that

$$
\begin{aligned}
& \tilde{\varepsilon}=\varepsilon L^{1 / \nu} \text { above } T_{c} \\
& \tilde{\varepsilon}=\varepsilon L^{1 / \nu^{\prime}} \text { below } T_{c}
\end{aligned}
$$

and

$$
\begin{aligned}
& s v=(2-\alpha) \\
& s v^{\prime}=\left(2-\alpha^{\prime}\right)
\end{aligned}
$$

Notice that if we assert that $\tilde{K}$ is a regular function of $\varepsilon, \tilde{\varepsilon}$, which is $\tilde{K}-K_{c}$, cannot have a discontinuous first derivative with respect to $\varepsilon$. Therefore, our assertion about the regularity 
of $K$ implies

$$
v=v^{\prime}
$$

which in turn implies $\alpha=\alpha^{\prime}$. It should be recognized that equation (15) is more dubious than the other assertions of this paper. Consequently, we hold this statement aside and do not use it in simplifying our further analysis.

More generally, when $h \neq 0$, we can equate the singular parts in $\varepsilon$ and $h$ on both sides of equation (10) and find

$$
f_{\operatorname{sing}}(\varepsilon, h)=\frac{1}{L s} f_{\operatorname{sing}}(\tilde{\varepsilon}, \tilde{h}) .
$$

This can only be true if $h$ is proportional to some power of $L$, i.e. if

$$
<\sigma>L^{-\psi}
$$

and then (16) and (14) can hold true if the singular part of $f(\varepsilon, h)$ obeys

$$
f_{\text {sing }}(\varepsilon, h)=\left\{\begin{array}{l}
\varepsilon^{2-\alpha} F(\varepsilon(s-\psi) v / h) \text { for } T>T_{c} \\
|\varepsilon|^{2-\alpha^{\prime}} F^{\prime}\left(|\varepsilon|(s-\psi) v^{\prime} / h\right) \text { for } T<T_{c}
\end{array}\right.
$$

This is Widom's homogeneity assertion [1] which we have now derived from our model.

If $\alpha=0$, and $f$ has the form (12b), then the last term on the right-hand side of equation (10) has a singular part

$$
\frac{\tilde{\varepsilon}^{2}}{L^{s}}[B-A \log \tilde{\varepsilon}]=\varepsilon^{2}\left[B-A \log \varepsilon-A \log L^{1 / \nu}\right]
$$

if we again employ (13) for this case. The extra term in $\log L^{1 / v}$ must be cancelled out by some other term on the right-hand side of (10). But since this term is regular in $\varepsilon$, the compensating term can be obtained by taking $f_{L}{ }^{(2)}$ in equation (2) to be

$$
f_{L}^{(2)}=A \log L^{1 / v}+\text { regular terms }
$$

or by a similar structure in $s L^{-s} f_{\text {int }}(\varepsilon, L)$. When $\alpha^{\prime}>0$, this term in (20) does not contribute to the leading singularity in $f(\varepsilon, h)$ for $T<T_{c}$ and consequently the analysis which led to (18) can go through just as before except that a term in $\varepsilon^{2} \log |\varepsilon|$ must be added to $F$ and $F^{\prime}$.

But, when $\alpha^{\prime}$ is also zero, the same term, (20), must cancel out $\log L$ terms both above and below $T_{c}$. The coefficient in front of this term cannot change at $T_{c}$ since $f_{L}$ is, by hypothesis, regular in $\varepsilon$. Then when $\alpha=\alpha^{\prime}=0$, and hence $\nu=\nu^{\prime}$ by (14), we conclude with Widom that

$$
A=A^{\circ}
$$

It is relevant to notice that this equality holds in the Onsager solution of the two dimensional 
Ising model [4]. It is interesting, but perhaps quite besides the point to notice that it is also true for the $\Lambda$-transition [5] of $\mathrm{He}^{4}$ and in at least one anti-ferromagnetic transition [6].

The arguments of Widom are designed to use the homogeneity of the singular part of $f(\varepsilon, h)$, as exhibited in equation (18), to derive relations between the average magnetization,

$$
\left.M \sim\langle\sigma\rangle \sim \frac{\partial f(\varepsilon, h)}{\partial h}\right|_{\varepsilon}
$$

the spin susceptibility

$$
\left.x \sim \frac{\partial\langle\sigma\rangle}{\partial h}\right|_{\varepsilon}
$$

and the parameters, $\alpha, \alpha^{\prime}, \nu$ and $\nu^{\prime}$ which we have already defined. If we define $\beta, \gamma, \gamma^{\prime}$ and $\delta$ in the conventional manner $[1,2]$

$$
\begin{aligned}
<\sigma> & =|\varepsilon|^{\beta} \quad \text { for } T<T_{c}, h=0 \\
\chi & =\varepsilon^{-\gamma} \quad \text { for } T>T_{c}, h=0 \\
\chi & =|\varepsilon|^{-\gamma^{\prime}} \text { for } T<T_{c}, h=0 \\
<\sigma> & =|h|^{1 / \delta} \text { for } \varepsilon=0
\end{aligned}
$$

it follows from (18) that

$$
\begin{aligned}
& \alpha^{\prime}+\beta(1+\delta)=2 \\
& \gamma^{\prime}+2 \beta+\alpha^{\prime}=2 \\
& \gamma^{\prime} / \gamma=\left(2-\alpha^{\prime}\right) /(2-\alpha) .
\end{aligned}
$$

Since the parameters in (25) are all experimental quantities, these relations could be checked if we could find an Ising model in nature.

In the course of this analysis, we also find that $\psi$ can be written in terms of experimental quantities as

$$
2 \psi=s-\gamma / v \text {. }
$$

This result is useful in the analysis in the spin-spin correlation function of equation (11), which equation can now be written as

$$
g\left(\varepsilon, h,\left|r-r^{\prime}\right|\right)=L^{-2 \psi} g\left(\varepsilon L^{1 / \nu}, h L^{s-\psi},\left|r-r^{\prime}\right| / L\right)
$$

for $T>T_{c}$. The right-hand side of (27) will only be independent of $L$ if $g$ is a homogeneous 
function of its arguments of the form

$$
\frac{1}{\left|r-r^{\prime}\right|^{2 \psi}} G\left(\varepsilon^{\nu(s-\psi)} / h,\left|r-r^{\prime}\right| \varepsilon^{\nu}\right) \text { for } T>T_{c}
$$

$g\left(\varepsilon, h,\left|r-r^{\prime}\right|\right)=$

$$
\frac{1}{\left|r-r^{\prime}\right|^{2} \psi} G^{\prime}\left(\varepsilon^{\nu^{\prime}(s-\psi)} / h,\left|r-r^{\prime}\right| \varepsilon^{\nu^{\prime}}\right) \text { for } T<T_{c}
$$

Consequently, the coherence length that we discussed at the beginning of this paper must be at $h=0$

$$
\xi=\left\{\begin{array}{cc}
\varepsilon^{-\nu} & \text { for } T>T_{c} \\
|\varepsilon|^{-\nu^{\prime}} & \text { for } T<T_{c}
\end{array}\right.
$$

and at $\varepsilon=0$

$$
\xi=|h|-(s-\psi)
$$

The result (29) makes contact with the notation of Widom [3] and Fisher [2] and permits us to identify $v$ and $v^{\prime}$ as experimental quantities. Therefore, equations (14a) and (14b) may be viewed as experimental relations.

There is one more experimental relation to be obtained. From (28) as $\varepsilon$ and $h$ go to zero, $g$ is proportional to $\left|\mathbf{r}-\mathbf{r}^{\prime}\right|-2 \psi$. This relation is conventionally [2] written as

$$
g\left(\varepsilon=0, h=0,\left|r-r^{\prime}\right|\right)=\frac{1}{\left|r-r^{\prime}\right|^{s-2+\eta}}
$$

so that, from $(26)$, we can write

$$
\gamma=(2-\eta) v
$$

which is our final experimental relation.

\section{Discussion and Comparison with Other Theoretical Results}

One can view the preceding work as developing relations among the nine "experimental" quantities $\alpha, \alpha^{\prime}, \beta, \gamma, \gamma^{\prime}, \delta, \nu, \nu^{\prime}$ and $\eta$. Equations (14a), (14b), (25a), (25b), (25c) and (31) are the six such relations that we consider to be direct consequences of our model. The relation (15), $v=v^{\prime}$, which implies $\alpha=\alpha^{\prime}, \gamma=\gamma^{\prime}$ is a somewhat more tenuous conclusion. With (15) included, we have seven relations among our nine parameters; if (15) is rejected there are only six such relations. In the first case, then, there are three free parameters; in the second two. 
None of these relations are new. Essam and Fisher [7] argued for (25b) on the basis of homogeneity considerations not totally different from those of Widom [1] who made full use of the homogeneity conjecture to get (25a), (25b), (25c) and (31) as well as (15). Besides, (31) can be viewed as a tautology: a definition of the coherence length. In this sense, the relation

$$
\gamma^{\prime}=(2-\eta) v^{\prime}:
$$

which follows from (31), (25c), and (14) can also be viewed as trivial.

There exists an alternative derivation of equation (14a) due to Pippard [8] and Ginsberg [9]. Imagine that we were in a situation of zero magnetic field in which the temperature lay just above $T_{c}$, i.e. $\varepsilon>0$. Then imagine that a temperature fluctuation occurred which took a region of side $X$ of the material into the ordered state. This would cost a free energy of the order of the volume of the region times the difference in free energy density between that at $T$ and that at $T_{c}$, i.e.

$$
X_{\varepsilon}^{s}(2-\alpha)
$$

But, the probability that such a fluctuation will occur is proportional to the exponential of the cost in free energy, divided by $k T_{c}$. Therefore, the maximum possible value of $X$, which is the coherence length, is given by setting (33) to be of order $k T_{c}$ or

$$
X_{\max } \sim \xi \sim \varepsilon^{-(2-\alpha) / s} \text {. }
$$

Therefore, we find at once $v=(2-\alpha) / s$.

Despite this simple derivation equations (14) are not unassailable. One could argue that, as $s-\infty$, we know that the molecular field approximation is valid. This gives $v=1 / 2, \alpha=0$, and (14) fails. But, it may well be that as $s-\infty$, the molecular field approximation becomes valid closer and closer to $T_{c}$; and for all finite $s$ there remains a region within a very small neighborhood of $T_{c}$ for which the molecular field approximation fails. Then (14) can still be true for all $s$, in the strict limit as $\varepsilon-0$.

Precisely this effect is expected to occur in the superconductor [8-10]. Here, the molecular field approximation (Landau-Ginzberg theory) is known to be valid until you get very close to $T_{c}$. This theory introduces a coherence length

$$
\xi_{\text {L.G. }}=|\varepsilon|^{-1 / 2} \xi_{0}
$$

where $\xi_{0}$ is a very large number $\sim 10^{4}$ lattice constants for a pure superconductor. Over most of the temperature range $\xi_{\text {L.G. }}$ is larger than the coherence length defined by (34). Then the molecular field theory remains valid because fluctuations in the order parameter are not important. But very close to $T_{c}$, the coherence length of (34) will surpass that of (35), and then the molecular field theory will fail. In this tiny temperature region about $T_{c}$, equations (14) are expected to be true if the analysis of reference [10] is correct.

To check the seven relations among physical parameters we compare with known results for two and three dimensional Ising models. A variety of numerical results have been obtained in both two and three dimensions through evaluations of the singularities of power series. These are reviewed in references [2,11-13]. In the two dimensional case there are also a variety of analytical results available [14-16] all based upon Onsager's solution [4]. For the two dimensional case, equations (28) would imply that the spin-spin correlation function is of the form 


$$
\left|r-r^{\cdot}\right|^{-1 / 4} H\left(\varepsilon\left|r-r^{\prime}\right|\right)
$$

when the magnetic field vanishes and $\left|r-r^{\prime}\right| \gg 1$. This result agrees with the conclusions of reference [16].

The second column of Table 1 lists the known values of the nine experimental parameters for the two dimensional Ising model. These all check exactly with widom's relations.

TABLE 1

\begin{tabular}{|c|c|c|c|c|c|}
\hline \multirow[b]{2}{*}{ Parameter } & \multicolumn{2}{|c|}{ Two dimensional case } & \multicolumn{3}{|c|}{ Three dimensional case } \\
\hline & Value & Reference & $\begin{array}{c}\text { Previously calculated } \\
\text { value }\end{array}$ & Reference & Fit to data \\
\hline$\alpha$ & 0 & 4 & $0.0 \leqslant \alpha \lesssim 0.2$ & 2 & 0.085 \\
\hline$\alpha^{\prime}$ & $\mathbf{0}$ & 4 & $0.0 \leqslant \alpha^{\prime} \lesssim 0.06$ & 2 & 0.085 \\
\hline$\beta$ & $1 / 8$ & 13 & $0.303 \leqslant \beta \leqslant 0.318$ & 2 & 0.332 \\
\hline$\gamma$ & $7 / 4$ & $2,11,16$ & $1.250 \pm 0.001$ & 2 & 1.250 \\
\hline$\gamma^{\prime}$ & $7 / 4$ & 2,16 & $1.23 \leqslant \gamma^{\prime} \leqslant 1.32$ & 2 & 1.250 \\
\hline$\delta$ & 15 & 13 & $5.2 \pm 0.15$ & 13 & 4.78 \\
\hline$v$ & 1 & 2 & $0.644 \pm 0.003$ & 2 & 0.638 \\
\hline$v^{\prime}$ & 1 & 16 & & 2 & 0.638 \\
\hline$\eta$ & $1 / 4$ & 14 & $0.060 \pm 0.007$ & 2 & 0.045 \\
\hline
\end{tabular}

The first column listed under the three dimensional case gives information which has been obtained via Pade and other numerical methods. The last column, labeled "Fit to data", gives a set of values for these parameters which agrees with all seven of our relations including the more doubtful statement $v=v^{\prime}$. This fit agrees with all the known numerical results to within about two and one-half standard deviations. These results indicate at least that the conclusions drawn from our model are not grossly inaccurate.

\section{Acknowledgnent}

I would like to thank Dr. P.C. Martin and Dr. Brian Josephson for very helpful comments. 


\section{References}

1. B. MIDOM, J. Chem. Phys. 43, 3898 (1965).

2. M. FISHER, J. Math. Phys. 5, 944 (1964).

3. B. WIDOM, J. Chem. Phys. 43, 3892 (1965).

4. For the two dimensional Ising model, the Onsager solution (L. ONSAGER, Phys. Rev. 65, 117, 1944 ) bears out equation (2).

5. M.J. BUCKINGHAM and W.M. FAIRBANK, in Progress in Low Temperature Physics III, (ed. C.J. Gorter), Ch. 3. North Holland, Amsterdam (1961).

6. J. SKALGO, Jr. and S. A. FRIEDBERG, Phys. Rev. Letters 13, 133 (1964).

7. J.W. ESSAM and M.E. FISHER, J. Chem. Phys. 39, 842 (1963).

8. A.B. PIPPARD, Proc. Roy. Soc. Lond. A216, 547 (1953).

9. D.M. GINSBERG and J.S. SHIER, to be published.

10. E. G. BATYEV, A.Z. PATASHINSKII and V.L. POKROVSKII, Zh. Eksp. Teor. Fiz. 46,2093 (1964). English translation in Sov. Phys.-JETP 19, 1412 (1964).

11. C. DOMB, Advanc. Phys. 9, 34, 35 (1960).

12. M.E. FISHER, J. Math. Phys, 4, 278 (1963).

13. M.E. FISHER, in Proceedings of the International Conference on Phenomena Near Phase Transitions, to be published.

14. B. KAUfMaN, Phys. Rev. 76, 1232 (1949); B. KAUfMAN and L. ONSAger, Phys. Rev. 76, 1244 (1949).

15. G.F. NEWELL and E.W. MONTROLL, Rev. Mod. Phys. 25, 353 (1953).

16. L. RADANOFF, to be published in Nuovo Cimento. 